\title{
Seeded growth of HgTe nanocrystals for shape control and their use in narrow infrared electroluminescence
}

Yoann Prado ${ }^{1}$, Junling Qu${ }^{1}$, Charlie Gréboval ${ }^{1}$, Corentin Dabard ${ }^{1}, 2$, Prachi Rastogi ${ }^{1}$, Audrey Chu¹, Adrien Khalili ${ }^{1}$, Xiang Zhen $\mathrm{Xu}^{2}$, Christophe Delerue ${ }^{3}$, Sandrine Ithurria ${ }^{2}$, Emmanuel Lhuillier ${ }^{{ }^{*}}$

${ }^{1}$ Sorbonne Université, CNRS, Institut des NanoSciences de Paris, INSP, F-75005 Paris, France. ${ }^{2}$ Laboratoire de Physique et d'Etude des Matériaux, ESPCI-Paris, PSL Research University, Sorbonne Université Univ Paris 06, CNRS UMR 8213, 10 rue Vauquelin 75005 Paris, France. ${ }^{3}$ Université de Lille, CNRS, Centrale Lille, ISEN, Université de Valenciennes, UMR 8520-IEMN, 59000 Lille, France

To whom correspondence should be sent: el@insp.upmc.fr

\section{Table of content}

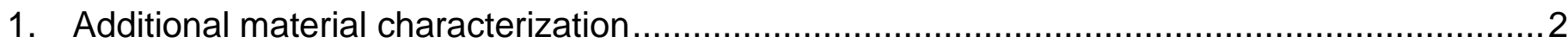

1.1. Evidence for coupling of nucleation and growth in Keuleyan's procedure ........................2

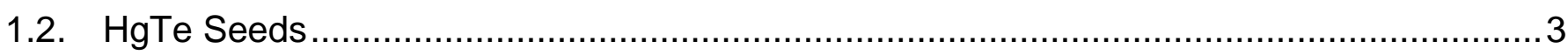

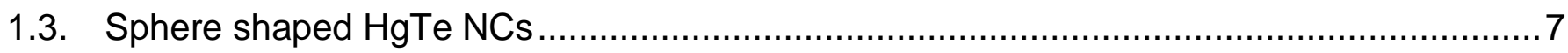

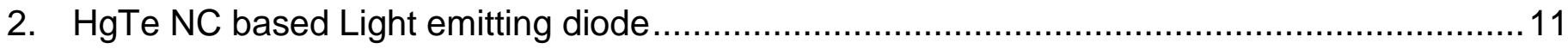

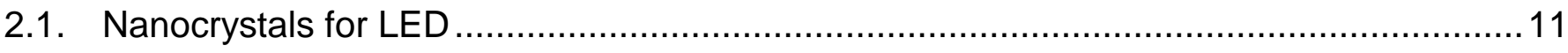

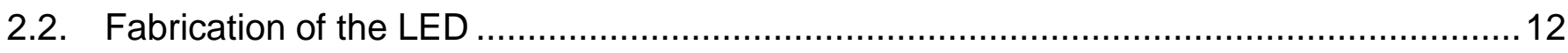

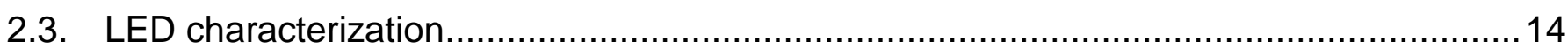

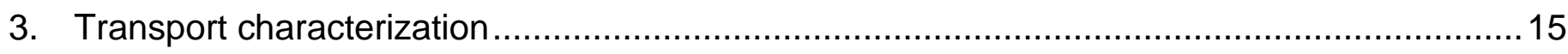

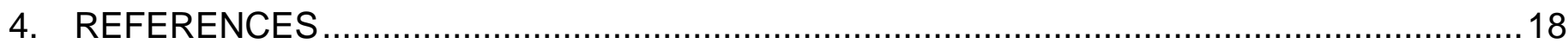


1. Additional material characterization

1.1. Evidence for coupling of nucleation and growth in Keuleyan's procedure
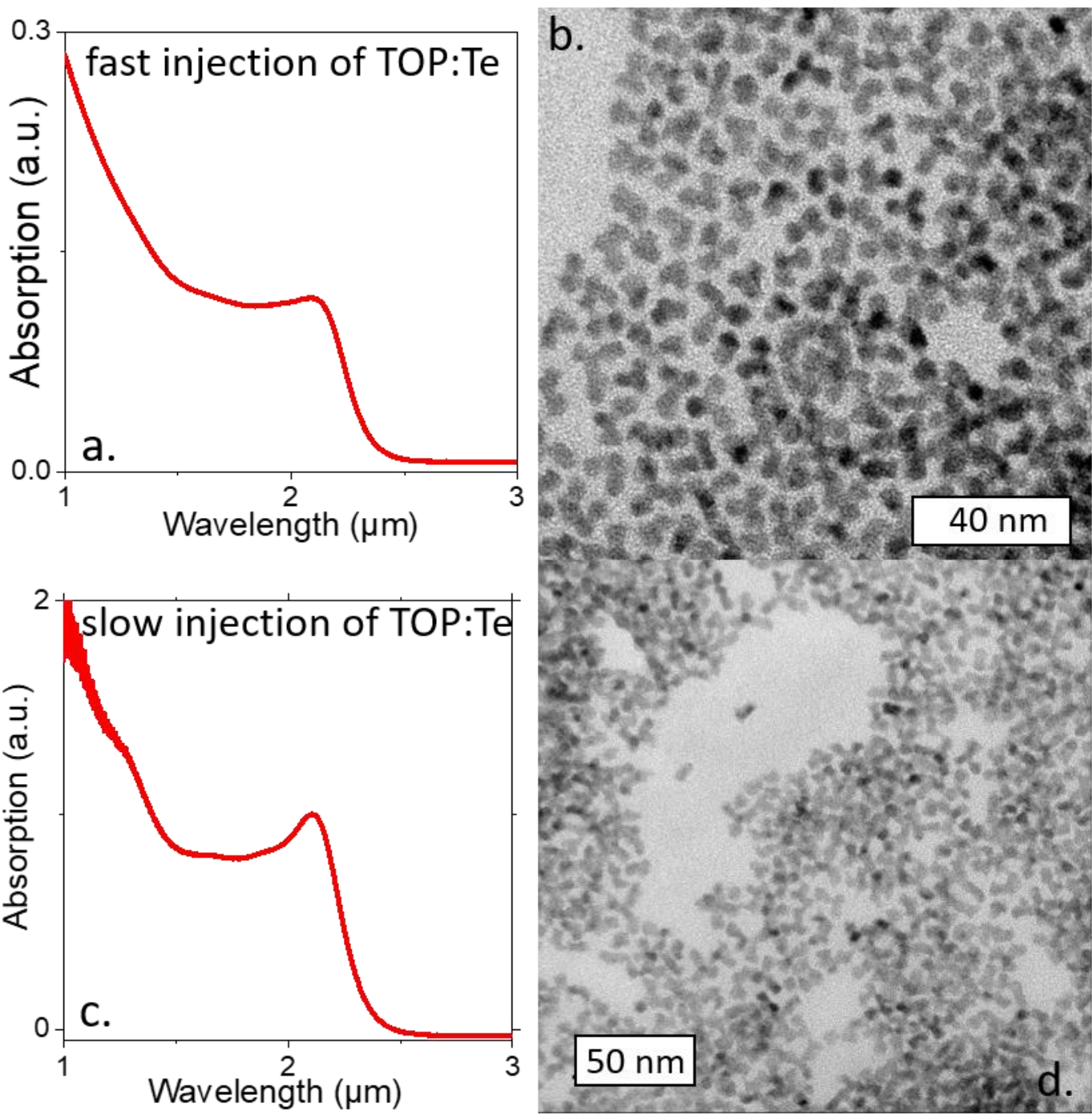

Figure $S 1$ a. Absorption spectrum for tripod HgTe NCs grown using the procedure depicted in ref' while the TOP:Te precursor is injected promptly ( $<3 s$ for 3 min of reaction). $b$ TEM image of the material whose spectrum is given in part a. c. Absorption spectrum for tripod HgTe NCs grown using the procedure depicted in ref ${ }^{1}$ while the TOP:Te precursor is injected slowly (25 s for 3 min of reaction).d. TEM image of the material which spectrum is given in part $c$. 


\section{Growth of the seed: In situ spectroscopic measurement of the seed growth}

In a glass vial, a) $81 \mathrm{mg}(0.3 \mathrm{mmol})$ of $\mathrm{HgCl}_{2}$, or b) $108 \mathrm{mg}(0.3 \mathrm{mmol})$ of $\mathrm{HgBr}_{2}$, or c) $135 \mathrm{mg}(0.3$ $\mathrm{mmol}$ ) of $\mathrm{Hgl}_{2}$ are added in $2.7 \mathrm{~mL}$ of oleylamine at RT. Then, $0.3 \mathrm{~mL}$ of TOP:Te $(1 \mathrm{M})$ is added and the mixture is mixed well with a pipette. To quench the reaction, $1.5 \mathrm{~mL}$ of a solution of DDT in toluene $(10 \% \mathrm{v} / \mathrm{v})$ is added. In this experiment, the solvents are not dried. Thus, residual water might be present in solvent and in air.
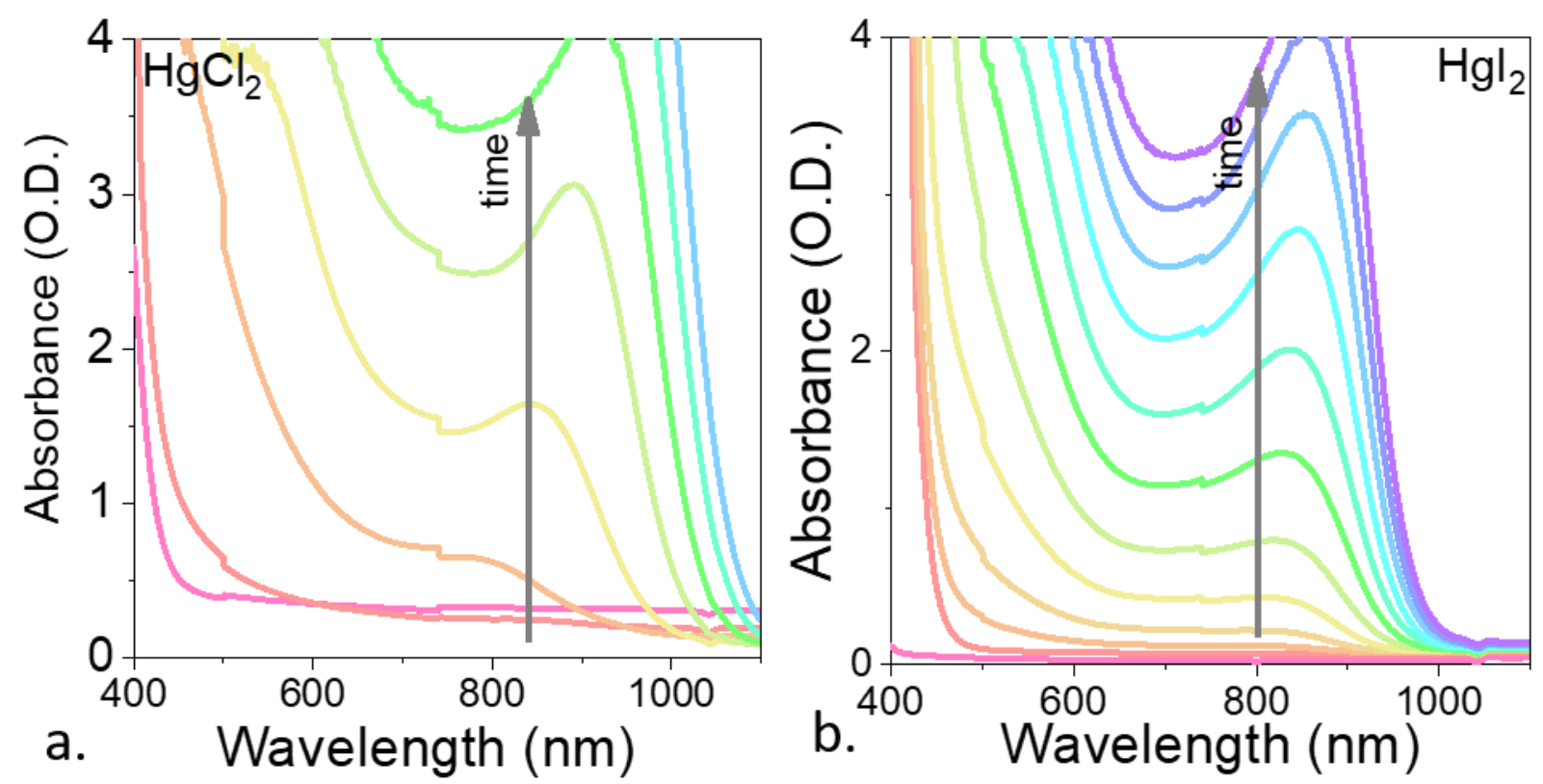

Figure $S 2$ a. UV-vis absorption spectra for a mixture of $\mathrm{HgCl}_{2}$ and TOP:Te in oleylamine at room temperature, leading to HgTe seeds. b. Same graph while using $\mathrm{Hgl}_{2}$ as precursor. 


\section{Effect of water on the seed growth}

Figure S 3 shows the effect of moisture of the seed formation. In presence of moisture the seed formation is clearly fasten
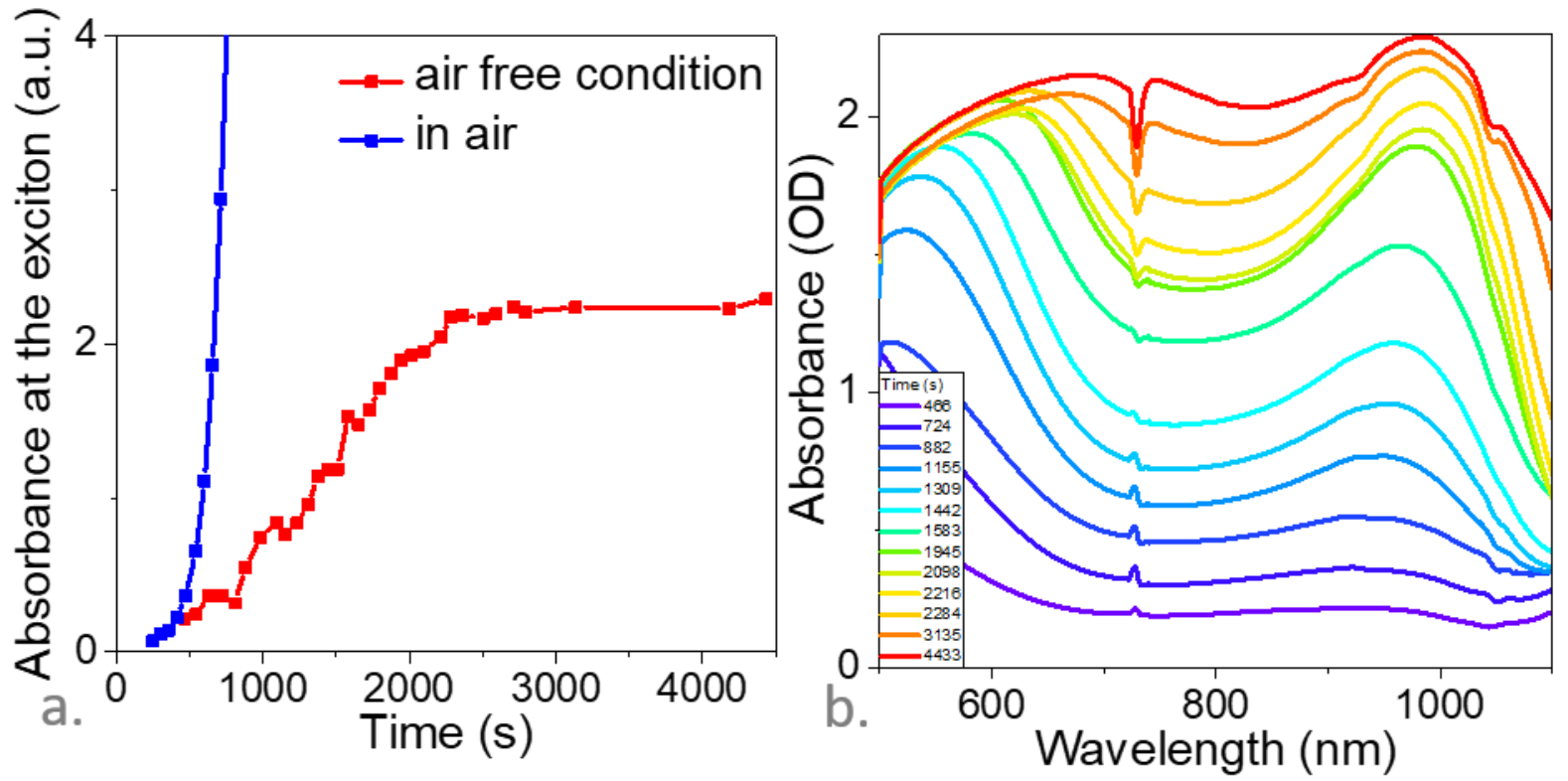

Figure $\mathrm{S} 3$ a. Absorption at the exciton as a function of time of the seeds (using $\mathrm{HgBr}_{2}$ as precursor) while conducting the reaction air free and in room condition b. UV-vis absorption spectra for a mixture of $\mathrm{HgBr}_{2}$ and TOP:Te (1:1 Hg:Te ratio) in oleylamine at room temperature, leading to $\mathrm{Hg} \mathrm{Te}$ seeds at different time after the mixing of the $\mathrm{Hg}$ and Te precursor.

To clarify the effect of water on the seed growth. We have performed seed growth under nitrogen (using non dried solvent) and while adding water. The amount of water is given in $\mathrm{Hg}$ molar equivalent, and the reaction is conducted under nitrogen. Clearly water fasten the reaction, see Figure S $4 \mathrm{a}$. The spectra of the seed are barely affected by the presence of water, see Figure S 4b. On the other hand, addition of water impacts the later use of the seed for the growth of sphere shape $\mathrm{HgTe}$ NC. Absorption spectrum for the sample containing 1 equivalent of water $(v s \mathrm{Hg})$ does not present the characteristic feature of the round shape HgTe, see Figure S 4c.

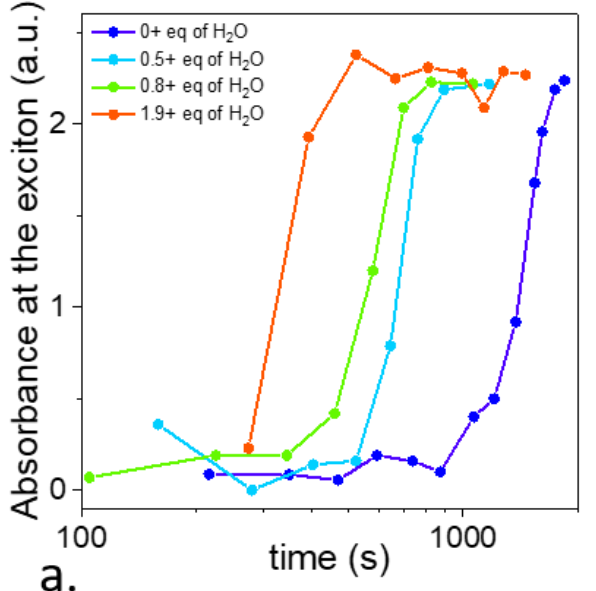

a.
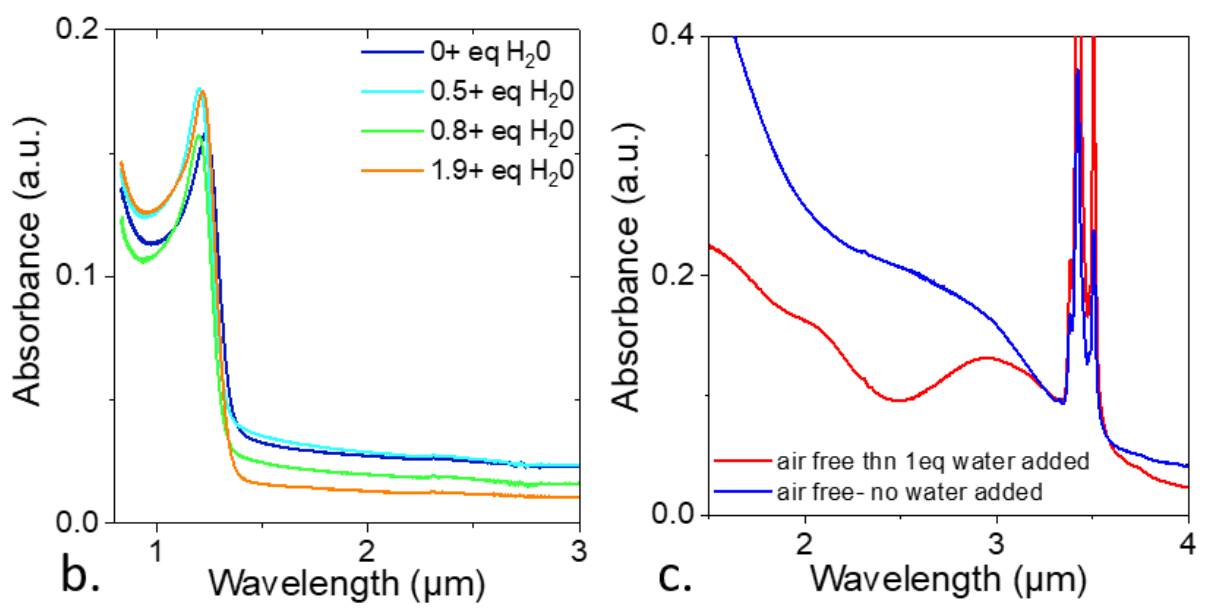
Figure $S 4$ a. Absorption at the exciton as a function of time for the seeds using $\mathrm{HgBr}_{2}$ as precursor, and while adding intentionally water at the beginning of the reaction. b. UV-vis absorption spectra for a mixture of $\mathrm{HgBr}_{2}$ and TOP:Te in oleylamine at room temperature, while various amount of water are added. Symbol + indicates that there might be water in the solvent. c. Absorption spectrum of the $\mathrm{HgTe} \mathrm{NC}$ obtained using the seeded path while water is only present are residue or intentionally added in the reaction medium

\section{Effect of Stoichiometry on the seeds}

The stoichiometry between $\mathrm{Hg}$ and $\mathrm{Te}$ seems to marginally affects the final seeds size, according to their absorption spectrum, see Figure S 5.

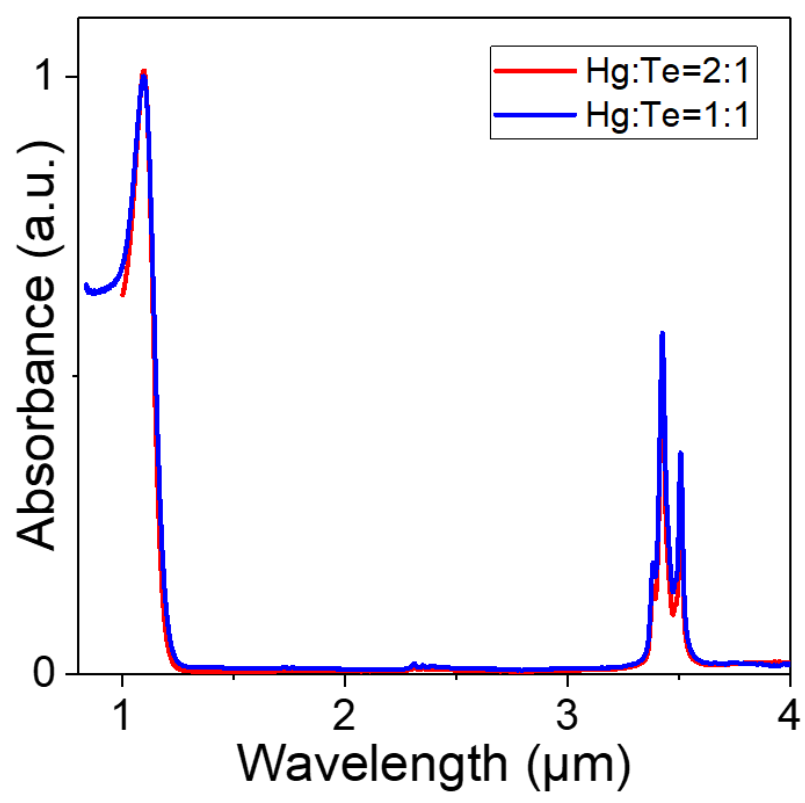

Figure S 5absorpotion spectra of the quenched $\mathrm{HgTe}$ seeds for two ratio of $\mathrm{Hg}: \mathrm{Te}$

\section{Structure of the seed:}

The seed crystalline nature is confirmed by XRD and high resolution TEM, see Figure $S 6$. The Xray diffractogram shows that the seeds grow with a zinc blende lattice. A series of narrow peaks at low diffraction angle suggests the formation of a lamellar phase where plane of $\mathrm{Hg}$ are spaced by double layer of oleylamine ${ }^{2}$. Using Scherrer law we can estimate the size of the particle $\left(\mathrm{L}=\frac{0.93 \lambda}{F W H M * \cos \theta}\right)=$ with $\lambda$ the wavelength of the $\mathrm{X}$-ray source $\left.(\mathrm{Cu} \mathrm{Ka}-0.154 \mathrm{~nm})\right)$, FWHM the full with at half maximum of the peak and $\theta$ a diffraction direction. We found $2.9 \mathrm{~nm}$. TEM leads to a consistent size with an estimate of $2.5 \mathrm{~nm}$. Assuming an average value for the size of $2.7 \mathrm{~nm}$, the number of atoms $\mathrm{Hg}$ and $\mathrm{Te}$ ) can be estimated around 300 per particle 

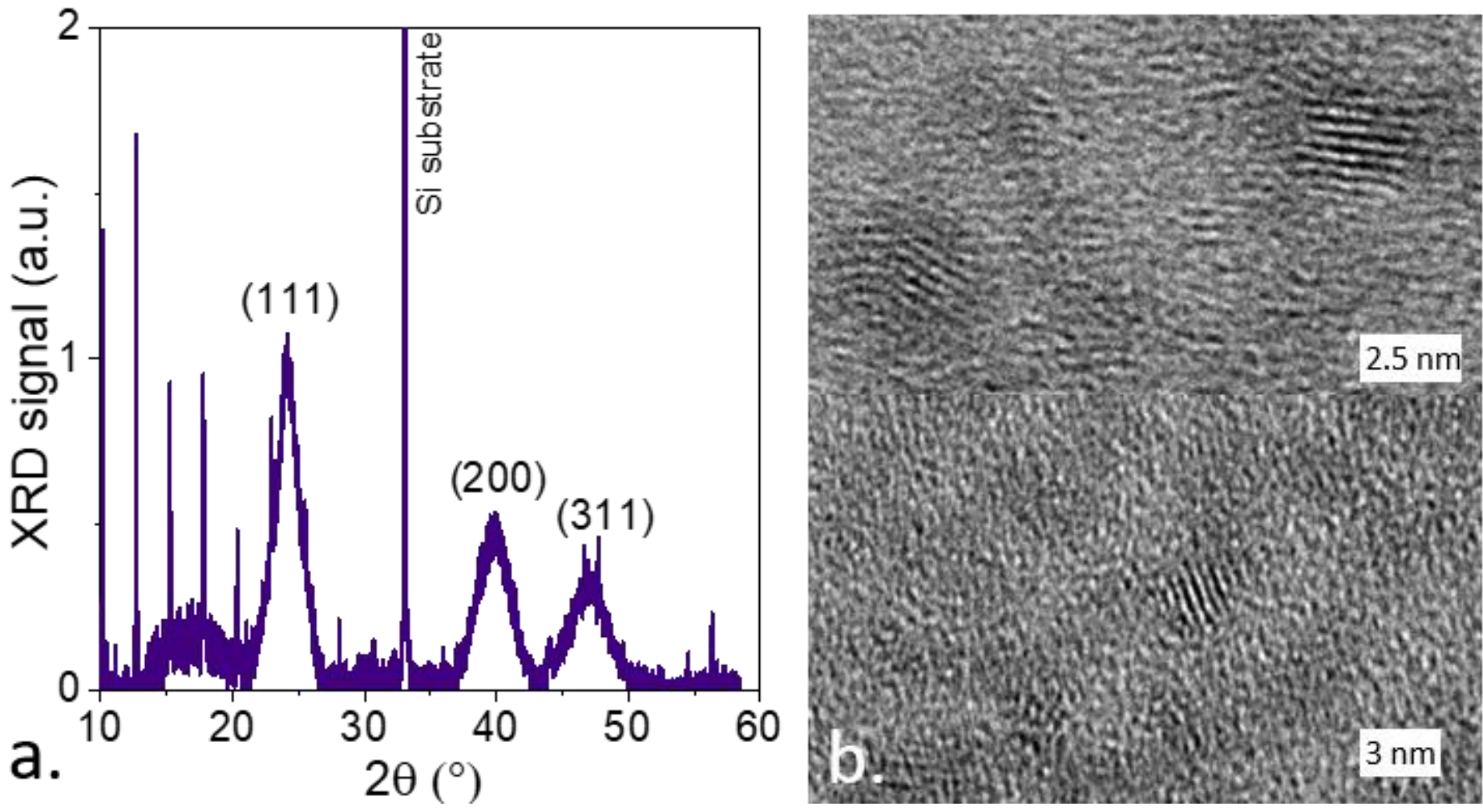

Figure S 6a. X-ray diffraction pattern from a film made of the HgTe seeds $b$. High resolution images of the seed.

Luminescence of the of the seed:

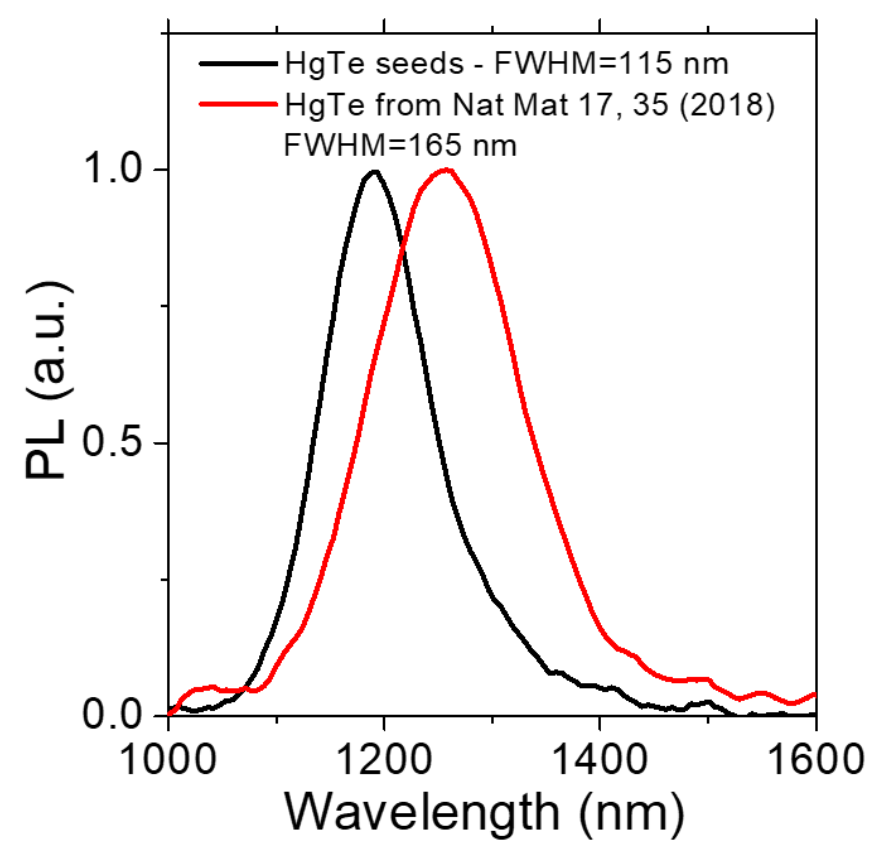

Figure S 7 Photoluminescence spectra of quenched HgTe seeds and small HgTe NCs obtained from the procedure of Geiregat et al. ${ }^{3}$ 


\subsection{Sphere shaped HgTe NCs}

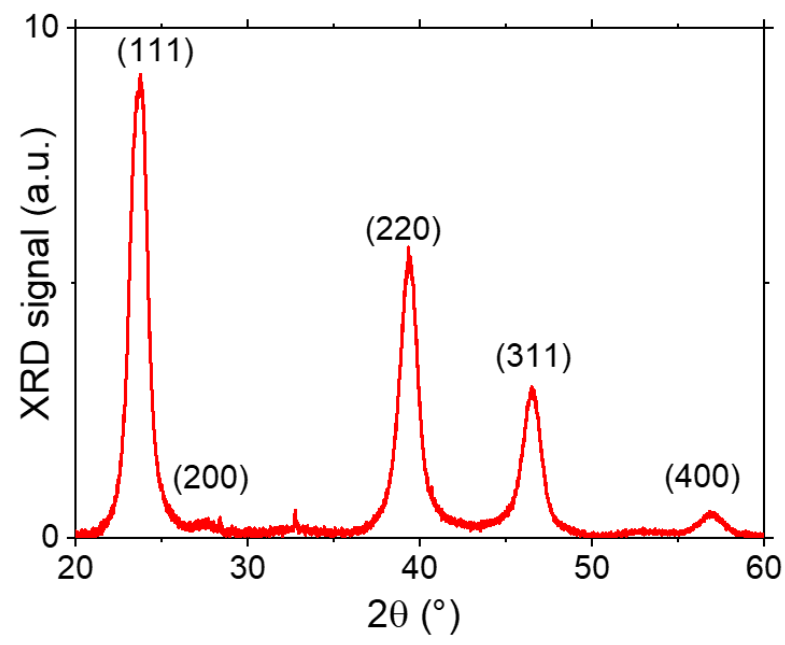

Figure S 8 X-ray diffraction pattern for a thin film of $\mathrm{HgTe}$ NCs obtained using the seeded procedure.
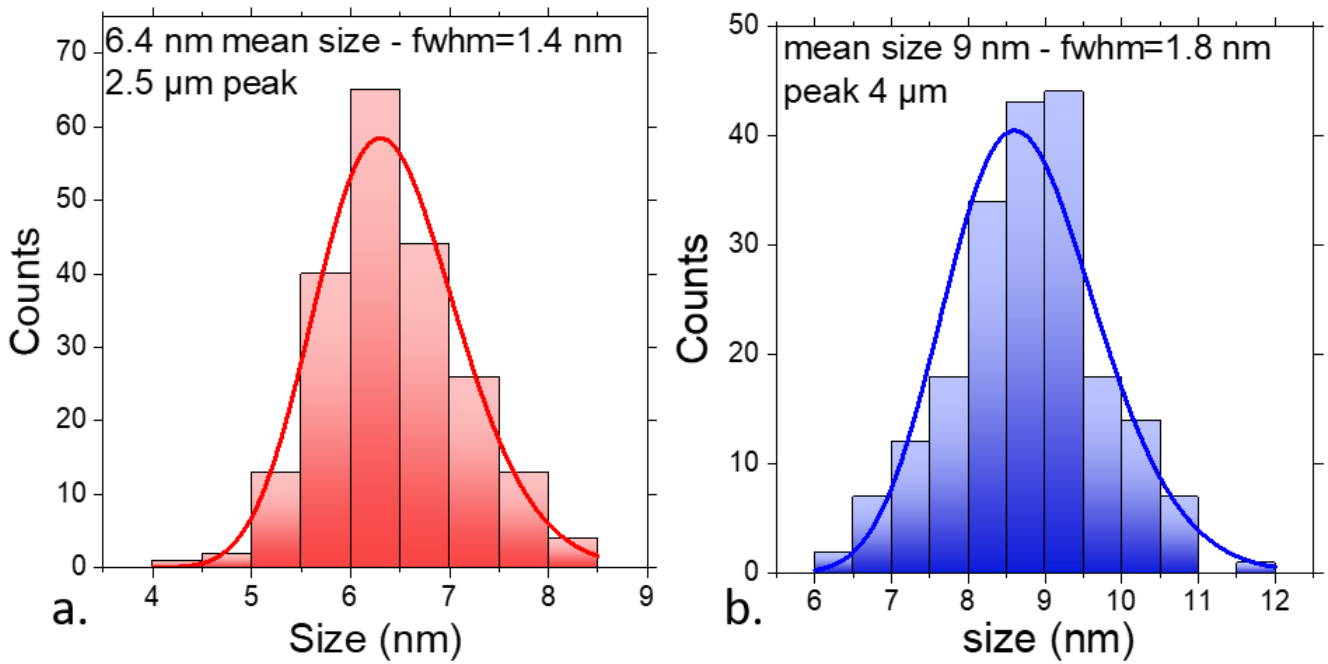

Figure S 9 Particle size distribution for sphere-shaped HgTe NCs with $2.5 \mu m$ peak absorption (a) and $4 \mu \mathrm{m}$ peak absorption (b).
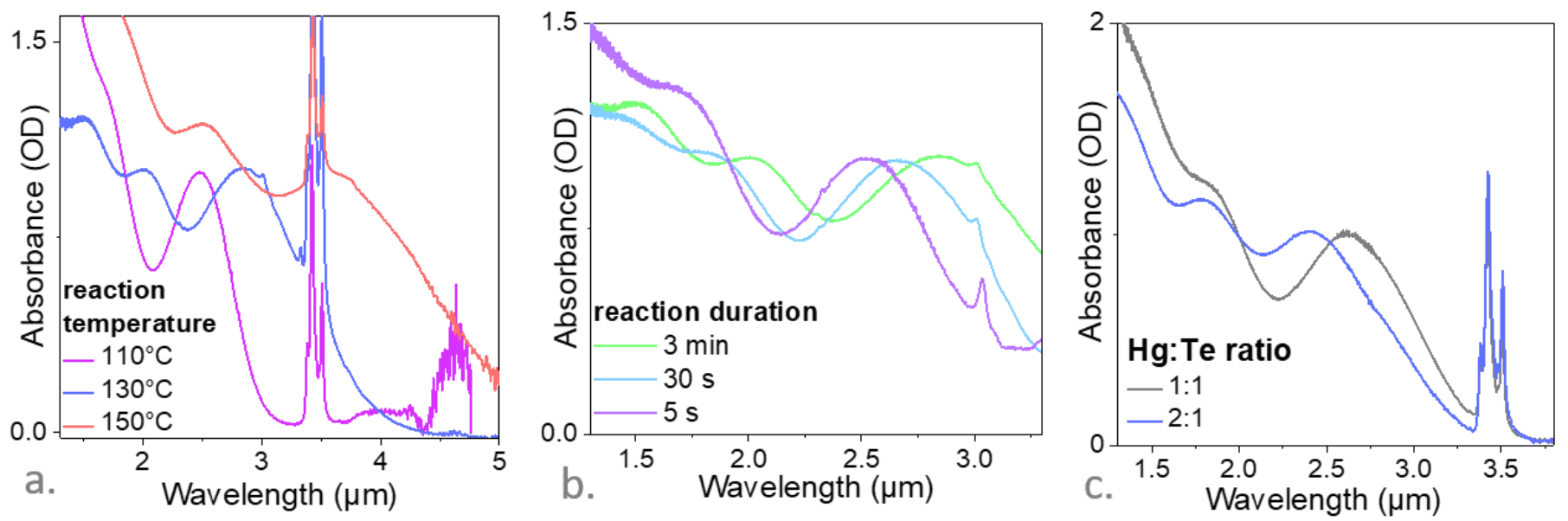

Figure S 10 Absorption spectra for sphere-shape HgTe NCs obtained from the seeded growth, as the temperature of the reaction is changed (a), as the duration of the reaction is changed (b) and as the stoichiometry of the reaction is tuned (c). 


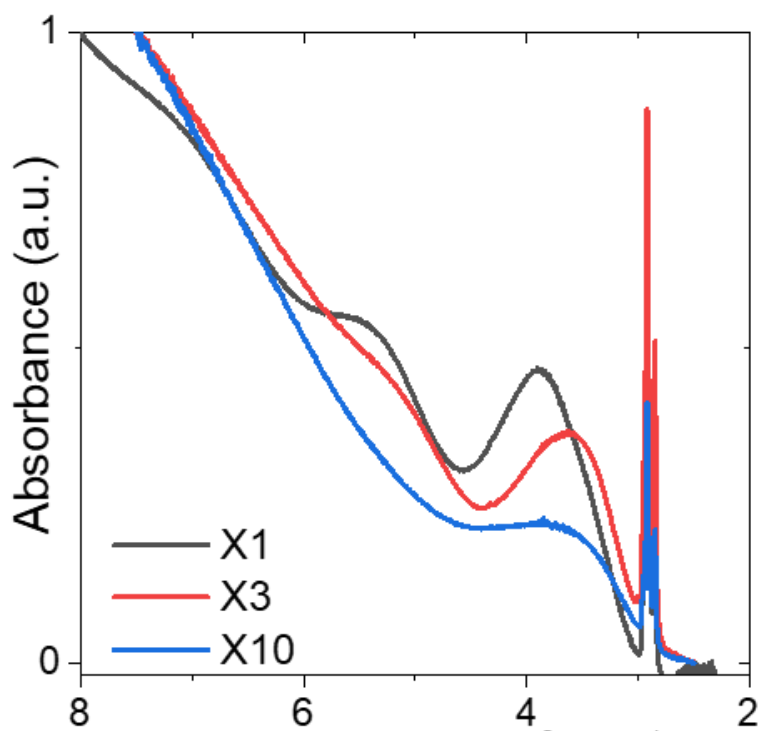

a. Wavenumber $\left(\times 10^{3} \mathrm{~cm}^{-1}\right)$

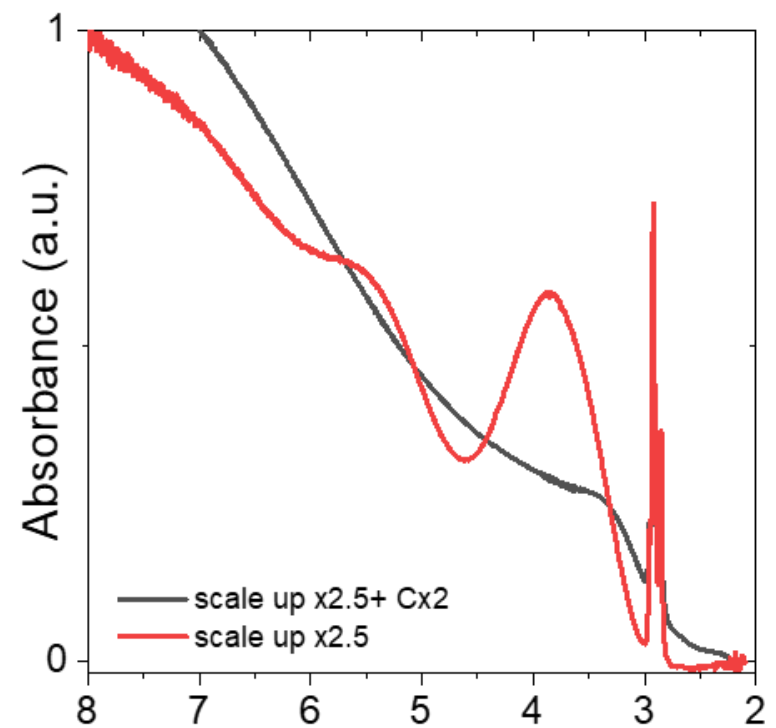

b. Wavenumber $\left(\times 10^{3} \mathrm{~cm}^{-1}\right)$

Figure S 11 a Absorption spectra for sphere-shape HgTe NCs for normal concentration and as the concentration is increased by factor 3 and 10. b Absorption spectra for sphere-shape HgTe NCs while the reaction is scaled up by a factor 2.5 for normal concentration and as the concentration is increased by a factor 2 . 


\section{Seeded growth vs particle rearrangement}

One important aspect relative to the mechanism of the growth is to determine the role of the free precursors in the growth of sphere-shaped particles. In the case of the procedure developed by Cassidy et al. ${ }^{4}$ for $\mathrm{CdSe}$, the growth occurs through a particle rearrangement. To unveil the mechanism at play in the case of $\mathrm{HgTe}$, we have also grown the nanoparticles after a cleaning step of the seeds. The seeds are precipitated and later reinjected in oleylamine (without free monomers). Figure $S 12$ a clearly shows that in this case, the obtained NCs are smaller (exciton shift is blue shifted from $2.6 \mu \mathrm{m}$ to $2 \mu \mathrm{m}$ ). TEM images (Figure $S 12 \mathrm{~b}$ and c) show that the shape is also affected since it is only possible to obtain sphere-shaped NC in presence of monomers.
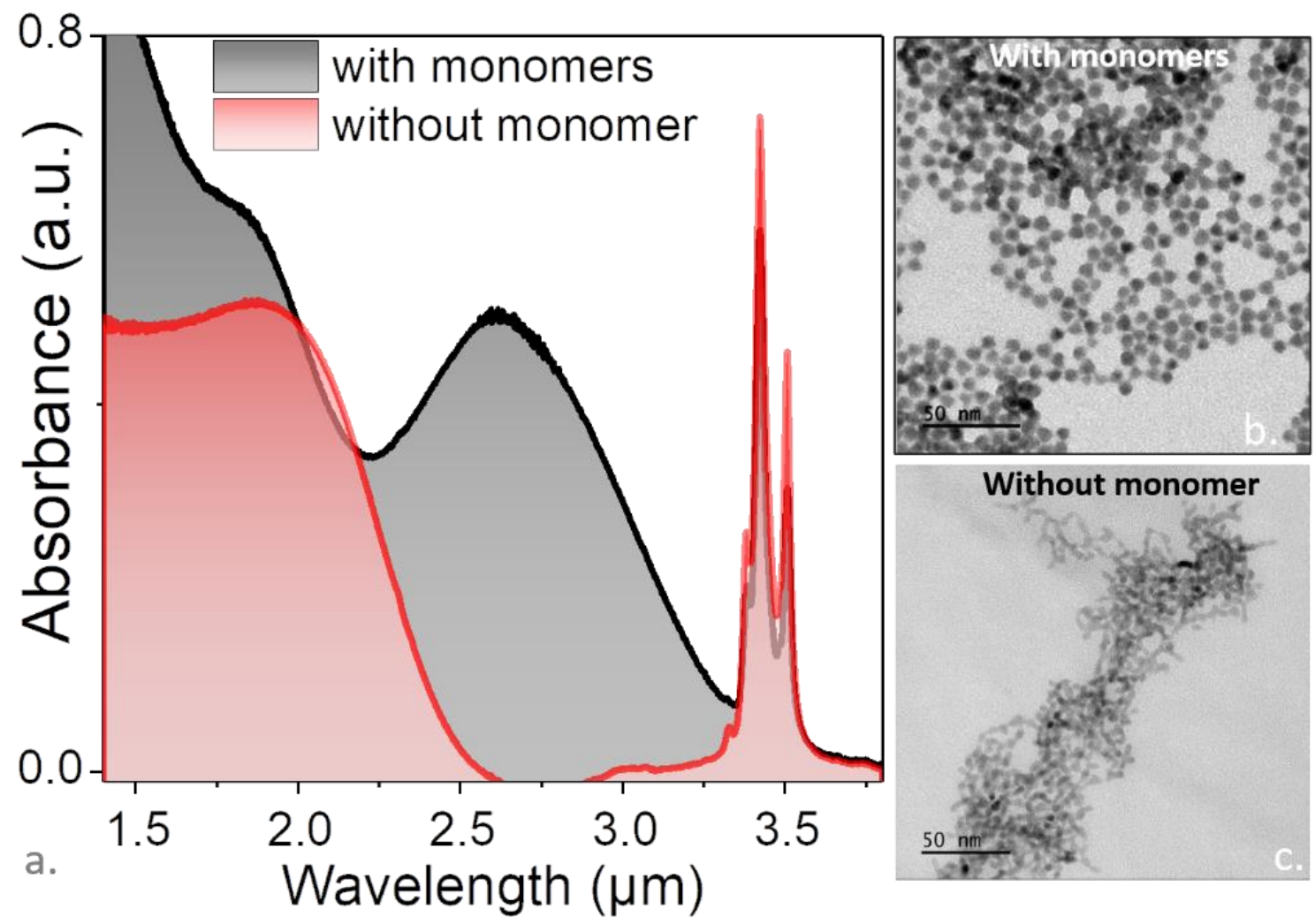

Figure S 12 a. Absorption spectra of the HgTe NCs obtained as the seeded growth is conducted with or without monomers. $b$. TEM image of the HgTe NCs obtained in presence of monomers. $c$. TEM image of the HgTe NCs obtained while the seeds have been cleaned to remove leftover of monomers. 
Absence of intraband absorption

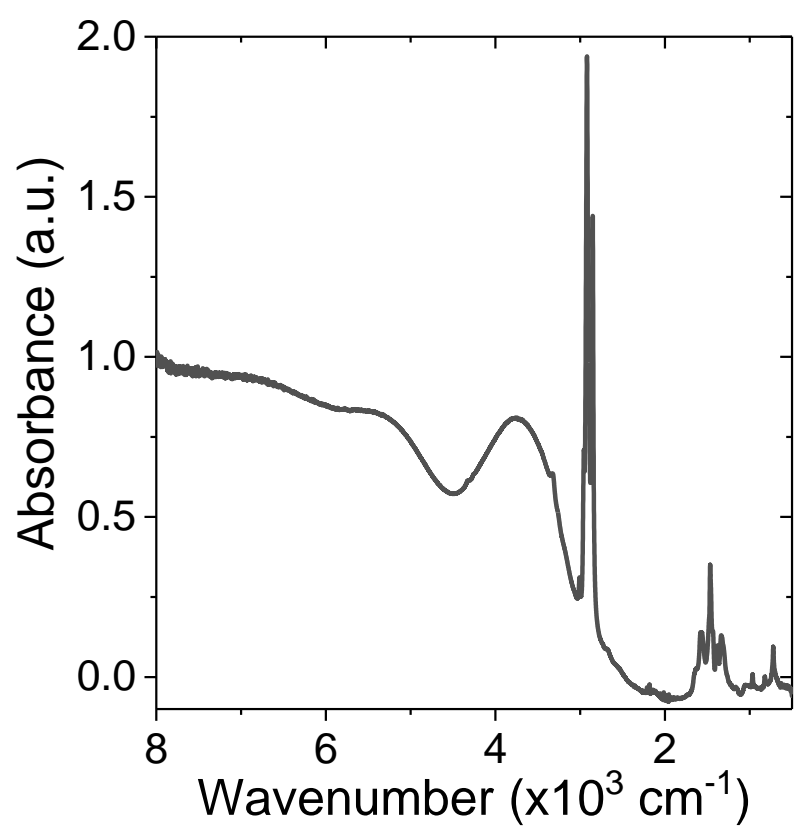

Figure S 13 mid-infrared spectrum for the sphere-shape HgTe NCs 


\section{HgTe NC based Light emitting diode}

\subsection{Nanocrystals for LED}

HgTe seeds for LED: In a $20 \mathrm{~mL}$ vial, $72 \mathrm{mg}$ of $\mathrm{HgBr}_{2}(0.2 \mathrm{mmol})$ was dissolved in $2.8 \mathrm{~mL}$ of oleylamine by sonication. Then $0.2 \mathrm{~mL}$ of TOP:Te $(1 \mathrm{M})$ was injected, and the mixture was stirred under ambient conditions for $45 \mathrm{~min} .3 \mathrm{~mL}$ of mixed solvent with $10 \%$ DDT in toluene $(\mathrm{v} / \mathrm{v})$ was injected to quench the reaction. The obtained products were first centrifuged to remove the unstable phase. The supernatant was then precipitated with methanol and redispersed in toluene for three times. During the last step of purification, the NCs were dried and redispersed in toluene at a concentration of $30 \mathrm{mg} \cdot \mathrm{mL}^{-1}$. The HgTe NCs were filtered with a $0.22 \mu \mathrm{m}$ PTFE filter before use. We observe a small redshift of the solution with time, see Figure $S 14$

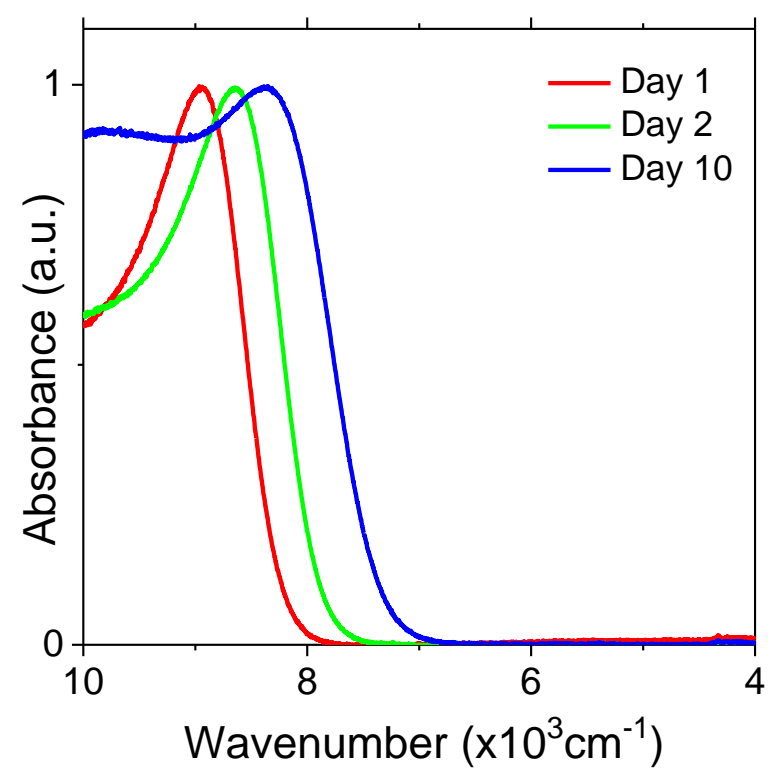

Figure S 14 absorption spectra of the HgTe seeds as a function of time

The synthesis of $\mathrm{ZnO}$ nanoparticles dispersed in ethanol: The procedure was taken from Dai et al ${ }^{5}$ with minor modifications. In flask $A, 1.32 \mathrm{~g}(6 \mathrm{mmol})$ of zinc acetate dihydrate was dissolved in $60 \mathrm{~mL}$ of DMSO by vigorous stirring. At the same time, $2 \mathrm{~g}(11 \mathrm{mmol})$ of TMAOH was dissolved with $20 \mathrm{~mL}$ of ethanol in flask B. Then the content of the two flasks were mixed and stirred for 24 hours under ambient conditions. The reaction mixture turned whitish during the first few seconds and became clear soon after. $\mathrm{ZnO}$ particles were first precipitated by ethyl acetate and redispersed in ethanol. $320 \mu \mathrm{L}$ of 2-ethanolamine were added to stabilize the nanoparticles before they were precipitated with ethyl acetate again and redispersed in $5 \mathrm{~mL}$ of ethanol. Finally, the $\mathrm{ZnO}$ nanoparticles in ethanol were filtered using a $0.22 \mu \mathrm{m}$ PTFE filter.

The synthesis of $\mathrm{ZnO}$ nanoparticles dispersed in chloroform: The synthesis procedure was taken from Pradhan et $\mathrm{al}^{6}$ with minor modifications. In a $100 \mathrm{~mL}$ three-neck flask, zinc acetate dihydrate $(0.98 \mathrm{~g})$ was dissolved in $42 \mathrm{~mL}$ of methanol under vigorous stirring and the temperature of the solution was set as $60^{\circ} \mathrm{C}$. In another vial, $0.49 \mathrm{~g}$ of KOH was dissolved in $22 \mathrm{~mL}$ of methanol. The $\mathrm{KOH}$ solution was added dropwise to the zinc acetate solution during $4 \mathrm{~min}$ at a constant 
temperature of $60^{\circ} \mathrm{C}$. The reaction mixture was kept under the same conditions for the next $2.5 \mathrm{~h}$. At the end of the reaction, the heating mantle was removed, and the solution cooled down to room temperature naturally. The reaction mixture was separated in two falcons and centrifuged at 6000 rpm for $2 \mathrm{~min}$. After discarding the supernatant, an equal volume of methanol was added to mix with the pellet, and another centrifugation was conducted. After three rounds of purification, the $\mathrm{ZnO}$ nanoparticles were dispersed in a mixed solvent with $2 \%$ butylamine in chloroform. The concentration of $\mathrm{ZnO}$ nanoparticles was set at $30 \mathrm{mg} \cdot \mathrm{mL}^{-1}$, and they were filtered through $0.22 \mu \mathrm{m}$ PTFE filter before use.

The synthesis of $\mathrm{PbS}$ nanoparticles: The procedure is inspired by Hines et $\mathrm{a} / \mathrm{l} 0.94 \mathrm{~g}$ of $\mathrm{PbO}$ were introduced in a $100 \mathrm{~mL}$ three-neck flask with $4 \mathrm{~g}$ of OA and $20 \mathrm{~g}$ of ODE. The flask was degassed under vacuum at $110{ }^{\circ} \mathrm{C}$ for 1 hour. Meanwhile, in an air-free glove box, a mixture of $720 \mu \mathrm{L}$ of (TMS) ${ }_{2} \mathrm{~S}$ and $20 \mathrm{~mL}$ of ODE was prepared. The atmosphere of the flask was switched to $\mathrm{N}_{2}$ and the temperature is set to $110^{\circ} \mathrm{C} .10 \mathrm{~mL}$ of the (TMS) $2 \mathrm{~S}$ solution were quickly injected and the solution turned dark. After $1 \mathrm{~min}$, the reaction was stopped by removing the heating mantle and promptly cooling of the flask by adding a mixture of hexane and oleic acid. The nanoparticles were then precipitated by ethanol. The formed pellet was redispersed in toluene. A second step of cleaning was conducted. Finally, the pellet was redispersed in toluene at a $50 \mathrm{mg} \cdot \mathrm{mL}^{-1}$ concentration. The solution was centrifuged to remove colloidally unstable materials. Finally, the solution was filtered on a $0.22 \mu \mathrm{m}$ PTFE filter. The obtained PbS NCs showed an exciton peak around $960 \mathrm{~nm}$.

\subsection{Fabrication of the LED}

ITO substrate patterning: ITO substrates $(10 \Omega / \square)$ with a thickness of $180 \mathrm{~nm}$ were cut into $15 \mathrm{~mm}$ $\times 15 \mathrm{~mm}$ pieces and cleaned by sonication in acetone for $5 \mathrm{~min}$. After sonication, the substrates were rinsed with acetone and isopropanol before dried completely with $\mathrm{N}_{2}$ flow. The substrates were further cleaned with $\mathrm{O}_{2}$ plasma for 5 min to remove organic residuals on the surface. After cleaning, TI-Prime and AZ 5214E photoresist were sequentially spin-coated on the surface of ITO substrates at the rate of $4000 \mathrm{rpm}$ for $30 \mathrm{~s}$ and baked at $110^{\circ} \mathrm{C}$ for $120 \mathrm{~s}$ and $90 \mathrm{~s}$, respectively. In the next stage, a mask aligner was used to expose the substrates to UV light for $20 \mathrm{~s}$ through a lithography mask ( $1 \mathrm{~mm}$ width). Photoresist was then developed using AZ 726 developer for $20 \mathrm{~s}$ before rinsed with deionized water and dried with $\mathrm{N}_{2}$ flux. After another 5 -minute plasma cleaning, the substrates were etched in a $25 \% \mathrm{HCl}$ (in water) bath for $10 \mathrm{~min}$ at $40^{\circ} \mathrm{C}$ before they were dipped immediately in deionized water. Finally, the lift-off was conducted in an acetone bath. Before using, the patterned ITO substrates were cleaned with acetone and isopropanol.

n-type ZnO layer deposition: On a patterned ITO substrate, the $\mathrm{ZnO}$ nanoparticles dispersed in ethanol was spin coated at $2000 \mathrm{rpm}$ for $1 \mathrm{~min}$. The $\mathrm{ZnO}$ film was then annealed at $250^{\circ} \mathrm{C}$ in air. The thickness of the film is determined to be around $100 \mathrm{~nm}$.

HgTe-ZnO emitting layer deposition: The same volume of $\mathrm{HgTe}$ solution in toluene and $\mathrm{ZnO}$ solution in chloroform were mixed and sonicated. Then the mixed solution was spin coated on the $\mathrm{ZnO}$ coated sample at a speed of $4000 \mathrm{rpm}$ for $30 \mathrm{~s}$. After the deposition, a solution of $5 \mathrm{mM} \mathrm{HgCl} 2$ in methanol $(13.6 \mathrm{mg}$ in $10 \mathrm{~mL}$ ) was casted onto the film and allowed to stay for $10 \mathrm{~s}$ before it was dried by spin coating at the same speed for $5 \mathrm{~s}$. The excess ligands on the film were rinsed twice 
with methanol and dried. In this way, three layers of $\mathrm{HgTe}-\mathrm{ZnO}$ were deposited and ligand exchanged, leading to an emitting layer of around $120 \mathrm{~nm}$.

p-type PbS layer deposition: The PbS NCs with an excitation peak of $980 \mathrm{~nm}$ was used as the hole transport layer. Inside an air-free glovebox, the PbS solution in toluene was diluted to $30 \mathrm{mg} \cdot \mathrm{mL}^{-}$ 1 and filtered with $0.22 \mu \mathrm{m}$ PTFE filter before use. Then a layer of PbS NCs was deposited on the top of the $\mathrm{HgTe}-\mathrm{ZnO}$ emitting layer by spin coating at $2500 \mathrm{rpm}$ for $15 \mathrm{~s}$. The ligand exchange was conducted with a $5 \mathrm{mM}$ (corresponding to $0.35 \% \mathrm{v} / \mathrm{v}$ ) solution of ethanedithiol (EDT) in acetonitrile. The EDT solution was drop casted to cover the PbS layer and allowed to stay for $45 \mathrm{~s}$ before dried with spinning. Subsequently, the film was rinsed twice with acetonitrile and then dried. Three layers of EDT capped PbS NCs were deposited and the thickness was measured to be $65 \mathrm{~nm}$.

Au top contact deposition: Inside a thermal evaporation chamber with a vacuum below $5 \times 10^{-6}$ mbar, $80 \mathrm{~nm}$ of Au was deposited onto the PbS layer through a shadow mask. The pixel area, which is defined as the overlap of the ITO and Au contact, is $1 \mathrm{~mm}^{2}$. 


\subsection{LED characterization}

Electroluminescence (EL) and photoluminescence (PL): $E L$ and PL spectra were obtained by a Flame NIR Spectrometer equipped with InGaAs detector from Ocean optics.

Current-Voltage-Radiance characteristics: The current-voltage-power of the LED device was collected with a Keithley K2400 source-meter unit and a PM100A power meter coupled with the S122C Ge detector from Thorlabs.

EQE and radiance can be extracted according to reference ${ }^{8}$. Assuming that the LED emission is Lambertian, the flux leaving the device can be directly described as $F_{\text {ext }}=\int_{0}^{\pi / 2} 2 \pi L_{0} \cos \theta \sin \theta d \theta=$ $\pi L_{0}$, with $L_{0}$ the flux per solid angle of light leaving the device in the forward direction. Since the solid angle from the photodetector to the light source is $\Omega=\frac{S_{1}}{l^{2}}$ with $S_{1}$ the area of the detector and $l$ the distance between the light source and detector, we can deduce that $L_{0}=\frac{P_{\text {det }}}{\Omega}=\frac{P_{\text {det }} l^{2}}{s_{1}}$ and $F_{\text {ext }}=$ $\frac{\pi P_{\mathrm{det}} l^{2}}{s_{1}}$. The number of photons emitted per second to the forward direction then can be calculated by $N_{P}=\frac{F_{e x t}}{h v}=\frac{\pi P_{\mathrm{det}} l^{2} \lambda}{s_{1} h c}$, with $\lambda$ the wavelength of the EL peak, $h$ the Plank's constant and $c$ the speed of light. The number of electrons injected per second can be obtained by $N_{e}=\frac{I}{e}$, with $I$ the current flow of the device. Thus, the EQE can be calculated as $=\frac{N_{p}}{N_{e}}=\frac{\pi P_{\mathrm{det}} l^{2} \lambda e}{s_{1} h c I}$, and the irradiance of the device $R=\frac{F_{e x t}}{\pi s_{2}}=\frac{\pi P_{\text {det }} l^{2}}{\pi s_{1} s_{2}}$, with $S_{2}$ the area of the pixel.

Operational lifetime measurement: During the lifetime measurement, the LED was driven at a constant current corresponding to a given initial radiance. The change of radiance (power) with time was collected with a power meter coupled with the S122C Ge detector. 


\section{Transport characterization}

Interdigitated electrodes for transport: The surface of a Siltronik dry oxide $\mathrm{Si} / \mathrm{SiO}_{2}$ wafer was cleaned by sonication in acetone. The wafer was rinsed with acetone, then isopropanol and dried with a $\mathrm{N}_{2}$ gun. A final cleaning was made using an $\mathrm{O}_{2}$ plasma for $5 \mathrm{~min}$. An adhesion promoter ( $\mathrm{TI}$ PRIME) was spin-coated onto the substrate and baked at $120^{\circ} \mathrm{C}$ for 2 min. AZ5214E was spincoated and baked at $110^{\circ} \mathrm{C}$ for $90 \mathrm{~s}$. The substrate was exposed under UV through a pattern mask for $1.5 \mathrm{~s}$. The film was then annealed at $125^{\circ} \mathrm{C}$ for $2 \mathrm{~min}$ in order to invert the resist. Then a $40 \mathrm{~s}$ flood exposure is performed. The resist was developed using a bath of AZ726 for $32 \mathrm{~s}$, rinsed in pure water and finally dried with $\mathrm{N}_{2}$. We then deposited $5 \mathrm{~nm}$ of chromium and $80 \mathrm{~nm}$ of gold using thermal evaporation. The lift-off was performed by dipping the film in acetone for 1 hour. The gold interdigitated electrodes were rinsed using isopropanol and dried with a $\mathrm{N}_{2}$ flow.

Electrolyte gating: $0.5 \mathrm{~g}$ of $\mathrm{LiClO}_{4}$ was mixed with $2.3 \mathrm{~g}$ of PEG $\left(\mathrm{Mw}=6 \mathrm{~kg} \cdot \mathrm{mol}^{-1}\right)$ in a glove box. The vial was heated at $170^{\circ} \mathrm{C}$ on a hot plate for $2 \mathrm{~h}$ until the solution turns clear. Then, the electrolyte solution was heated to $100^{\circ} \mathrm{C}$ and brushed on the top of the $\mathrm{HgTe}$ film.

CQD ink preparation: An exchange solution was prepared by dissolving $15 \mathrm{mg}$ of $\mathrm{HgCl}_{2}$ in $9 \mathrm{~mL}$ DMF and $1 \mathrm{~mL}$ of 2, mercaptoethanol. Then $0.5 \mathrm{~mL}$ of HgTe CQDs at $50 \mathrm{mg}^{-\mathrm{mL}^{-1}}$ in toluene was mixed with $0.5 \mathrm{~mL}$ of exchange solution. $1 \mathrm{~mL}$ of DMF was added to prevent precipitation. $5 \mathrm{~mL}$ of hexane was added and phase separation was observed. Hexane phase was discarded and two additional cleaning were performed. $2 \mathrm{~mL}$ of toluene was then added as bad solvent to precipitate the particles. After centrifugation the supernatant was discarded and the particles were dried under vacuum for $20 \mathrm{~min}$. Finally, $60 \mu \mathrm{L}$ of fresh DMF was added, leading to a colloidally stable ink.

Film deposition for transport: $3 \mu \mathrm{L}$ of the previously prepared ink was deposited onto interdigitated electrodes and spin coated at $2000 \mathrm{rpm}$ for $90 \mathrm{sec}$.

Electrolyte FET: Once electrolyte gate was deposited onto the CQD film, the device was connected to the two channels of a Keithley 2634B. The first channel was used to apply a drain source bias and measure the flowing current and the second one was used to apply the gate bias.

Backgate $\mathrm{Si} / \mathrm{SiO}_{2} \mathrm{FET}$ : The sample was placed in a pulsed tube cryostat under secondary vacuum. The temperature was regulated by a Lakeshore 335. The device was connected to the two channels of a Keithley 2634B. The first channel was used to apply a drain source bias and measure the flowing current and the second one was used to apply the gate bias. 

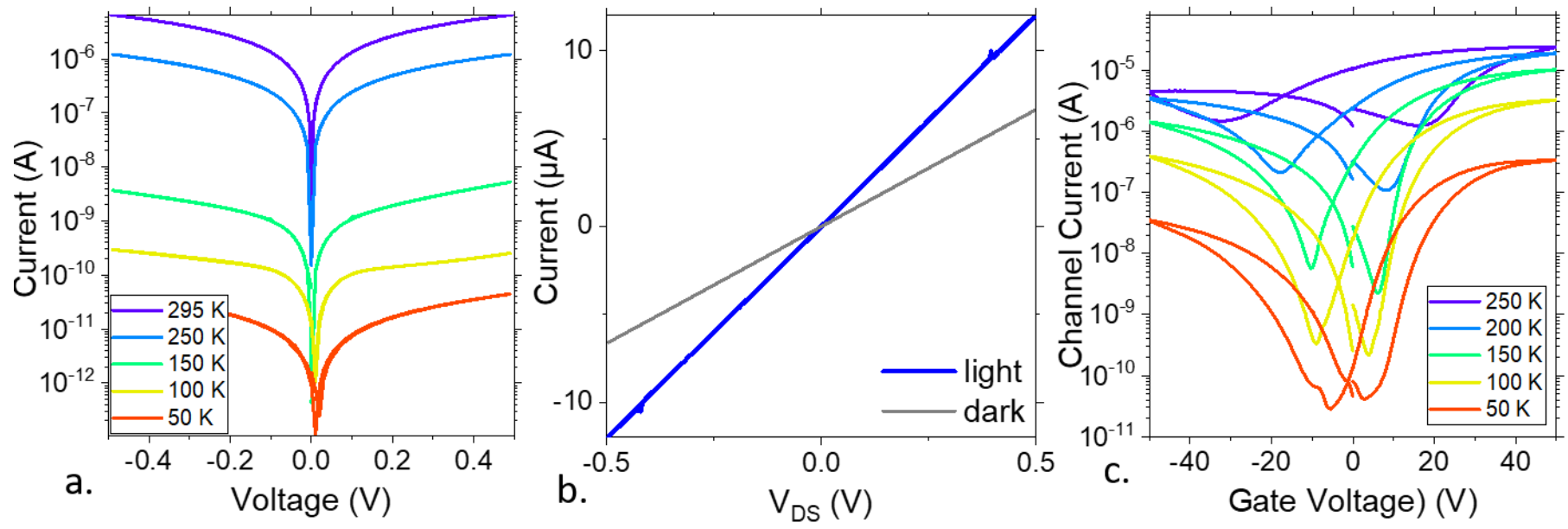

Figure $S 15$ a. Dark I-V curves at different temperature for thin films made of sphere-shaped HgTe NCs. b. I-V curves at room temperature in dark condition and under illumination $(\lambda=1.55 \mu \mathrm{m}, 2 \mathrm{~mW}$ laser diode power) for thin films made of sphere-shaped HgTe NCs. C. Transfer curves (drain current as a function of gate bias) at different temperatures for thin films made of sphere-shaped HgTe NCs with mercaptoethanol- $\mathrm{HgCl}_{2}$ surface chemistry. The gate is $\mathrm{SiO}_{2}$.

Responsivity measurement: A $1.55 \mu \mathrm{m}$ laser was electrically chopped at various frequencies and shone on the sample. The drain-source and the gate-source voltages are set by a Keithley K2634b. A DLPCA transimpedance amplifier was connected on the drain branch of the circuit to amplify the current before sending it to a Tektronix GHz scope. The time resolved photocurrent was then saved from the scope. The power of the laser was measured using a Thorlabs PM100A power meter equipped with a wide range detector.

Spectral response measurement: We use a thermo Fischer FTIR as interferometer. The white light source of the FTIR was fed into the interferometer. $\mathrm{A} \mathrm{CaF}_{2}$ beam splitter was used and the light finally shone on the sample. The sample was biased using a channel of a Keithley 2634b. The current was then amplified by a Femto DLPCA-200, and fed back to the electronic of the FTIR. Photocurrent spectra were then normalized by the gain of the amplifier used for each measurement.

Noise measurement. Current from the device (at $0.5 \mathrm{~V}$ bias, kept in the dark) is amplified by a Femto DLPCA-200, then fed into a SRS SR780 signal analyzer. Measurement is performed in air at room temperature 


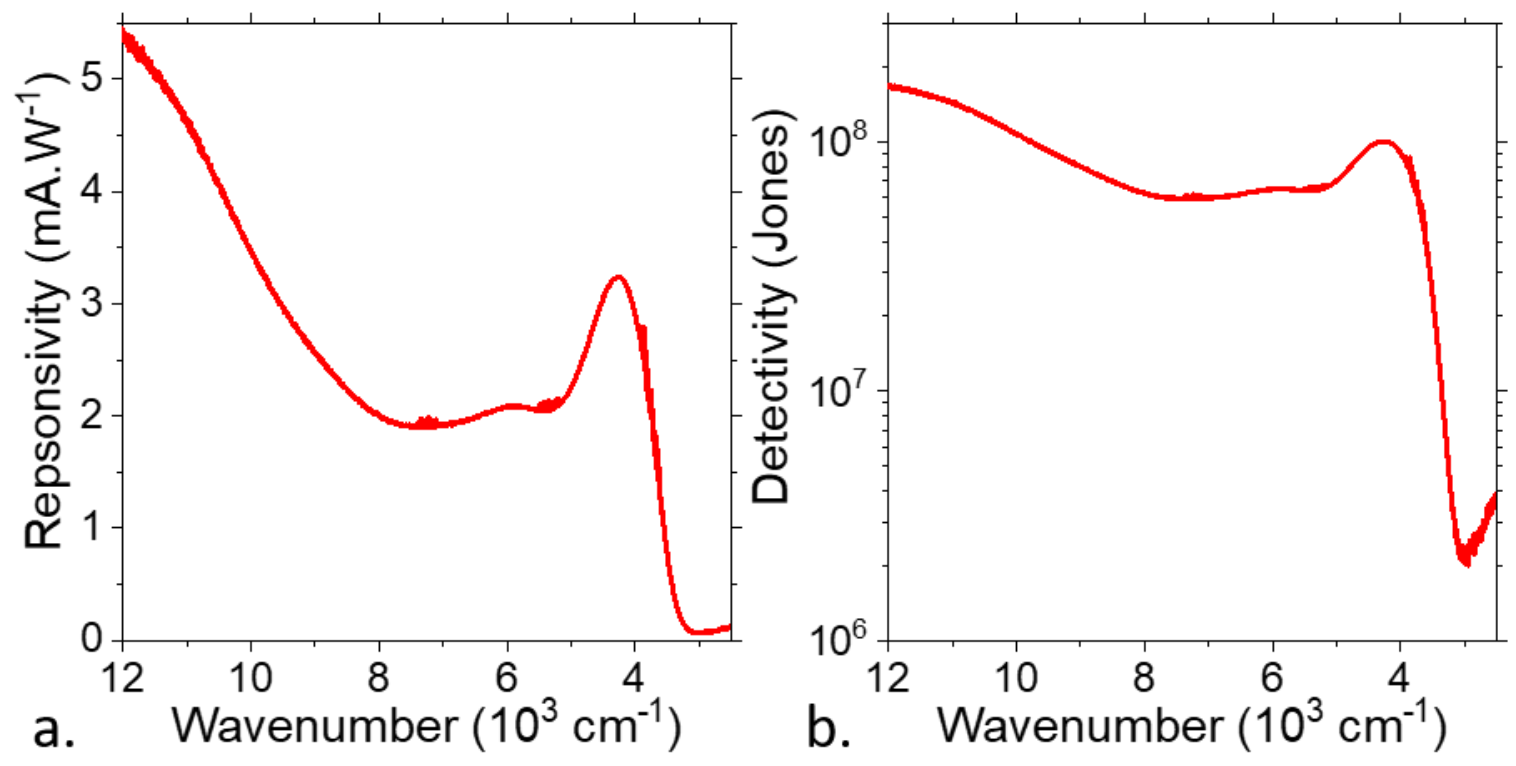

Figure $S 16$ responsivity (a.) and specific detectivity (b.) under $0.5 \mathrm{~V}$ as a function of signal wavenumber for a thin film made of sphere-shaped HgTe NCs 


\section{REFERENCES}

(1) Keuleyan, S.; Lhuillier, E.; Guyot-Sionnest, P. Synthesis of Colloidal HgTe Quantum Dots for Narrow Mid-IR Emission and Detection. J. Am. Chem. Soc. 2011, 133, 16422-16424.

(2) Goubet, N.; Thomas, M.; Gréboval, C.; Chu, A.; Qu, J.; Rastogi, P.; Chee, S. S.; Goyal, M.; Zhang, Y.; Xu, X. Z.; Cabailh, G.; Ithurria, S.; Lhuillier, E. Near- To Long-Wave-Infrared Mercury Chalcogenide Nanocrystals from Liquid Mercury. J. Phys. Chem. C 2020, 124, 84238430.

(3) Geiregat, P.; Houtepen, A. J.; Sagar, L. K.; Infante, I.; Zapata, F.; Grigel, V.; Allan, G.; Delerue, C.; Van Thourhout, D.; Hens, Z. Continuous-Wave Infrared Optical Gain and Amplified Spontaneous Emission at Ultralow Threshold by Colloidal HgTe Quantum Dots. Nat. Mater. 2018, 17, 35-42.

(4) Cassidy, J.; Ellison, C.; Bettinger, J.; Yang, M.; Moroz, P.; Zamkov, M. Enabling Narrow Emission Line Widths in Colloidal Nanocrystals through Coalescence Growth. Chem. Mater. 2020, 32, 7524-7534.

(5) Dai, X. L.; Zhang, Z. X.; Jin, Y. Z.; Niu, Y.; Cao, H. J.; Liang, X. Y.; Chen, L. W.; Wang, J. P.; Peng, X. G. Solution-Processed, High-Performance Light-Emitting Diodes Based on Quantum Dots. Nature 2014, 515, 9-6.

(6) Pradhan, S.; Di Stasio, F.; Bi, Y.; Gupta, S.; Christodoulou, S.; Stavrinadis, A.; Konstantatos, G. High-Efficiency Colloidal Quantum Dot Infrared Light-Emitting Diodes via Engineering at the Supra-Nanocrystalline Level. Nat. Nanotechnol. 2019, 14, 72-79.

(7) Hines, M. A.; Scholes, G. D. Colloidal PbS Nanocrystals with Size-Tunable Near-Infrared Emission: Observation of Post-Synthesis Self-Narrowing of the Particle Size Distribution. Adv. Mater. 2003, 15, 1844-1849.

(8) Chen, Z. Q.; Ding, F.; Bian, Z. Q.; Huang, C. H. Efficient Near-Infrared Organic Light-Emitting Diodes Based on Multimetallic Assemblies of Lanthanides and Iridium Complexes. Org. Electron. 2010, 11, 369-376. 